\title{
Microsporogenesis in a Citrus interspecific tetraploid somatic hybrid and its fusion parents
}

\author{
SERGIO FATTA DEL BOSCO*, NICASIO TUSA \& CLARA CONICELLA $\dagger$ \\ CNR-CMGA, Centro di Studio per il Miglioramento Genetico degli Agrumi. C.N.R., Viale delle Scienze - 90128 \\ Palermo, Italy and $\uparrow C N R-I M O F$, Istituto per il Miglioramento Genetico delle Piante da Orto e da Fiore, \\ Via Università 133-80055 Portici, Italy
}

\begin{abstract}
Microsporogenesis was analysed in a tetraploid somatic hybrid (SH) $(2 n=4 x=36)$ of Citrus and its diploid fusion parents $(2 n=2 x=18)$, Valencia sweet orange $(C$. sinensis L. Osbeck) and Femminello lemon (C. limon L. Burm. f.). Intergenomic pairing between lemon and orange occurred in the somatic hybrid which showed multivalent chromosome associations in diakinesis, although one quadrivalent was definitely because of a reciprocal translocation present in Valencia. The behaviour of univalents was variable in the somatic hybrid and its parents. In the somatic hybrid and Valencia, the univalents preferentially formed micronuclei and polyads whereas, in Femminello, they were generally enclosed in a nucleus although distributed randomly. The somatic hybrid showed a rate of pollen stainability of $64 \%$ and germinability of $41 \%$. The chromosomally unbalanced pollen from the tetraploid SH was presumed viable and able to fertilize because different nuclear DNA contents were found in the back-cross progeny. Moreover, meiotic nuclear restitution mechanisms, which could be mainly dependent on the abnormal orientation of the spindles in meiosis II, are described.
\end{abstract}

Keywords: lemon, meiosis, orange, somatic hybridization, unreduced pollen.

\section{Introduction}

Cultivar development in Citrus by conventional breeding methods has been limited by its complex reproductive biology (Swingle \& Reece, 1967; Grosser \& Gmitter, 1990a). Somatic hybridization was applied successfully in Citrus for combining complementary diploid elite scion varieties (Grosser \& Gmitter, 1990a, 1990b). Allotetraploid somatic hybrids of Citrus do not have the potential for direct use as scions but, if fertile, can be used as pollen parents in interploid sexual hybridization to generate new and improved seedless triploid Citrus varieties (Grosser \& Gmitter, 1990a). However, the relationship between the genomes of the parental species has great influence on determination of the process of chromosome pairing and recombination and thus the extent of meiotic irregularities and viability of the gametes (De Jong et al., 1993). Indeed, somatic hybrids generally have a lower fertility, which is a major problem in sexual reproduction (Pijnacker et al., 1992).

*Correspondence: E-mail: sfatta@unipa.it

(C) 1999 The Genetical Society of Great Britain.
The present study was conducted in an interspecific tetraploid Citrus somatic hybrid between Valencia sweet orange (C. sinensis L. Osbeck) and Femminello lemon $(C$. limon $\mathrm{L}$. Burm. f.) that was produced in an effort to combine the good quality and performance of Femminello lemon with the cold hardness and tolerance to 'mal secco' [a systemic fungal disease caused by Phoma tracheiphila (Petri) Kantsh et Ghik] from Valencia orange (Tusa et al., 1990). The somatic hybrid Valencia $(+)$ Femminello has already been used in interploid sexual hybridization with diploid lemon. Cytofluorimetric analysis of nuclear DNA content revealed variation in the ploidy level among the seedlings of the back-cross progeny which was expected to be uniformly triploid (Tusa et al., 1996). However, successful introgression of useful Valencia traits into the back-cross lemon offspring can be guaranteed by meiotic recombination between homologous chromosomes in the somatic hybrid. Therefore, in this work, meiotic behaviour was investigated in the tetraploid somatic hybrid to determine the level of intergenomic recombination and to identify the cytological mechanisms underlying the ploidy variation in the back-cross progeny. 


\section{Materials and methods}

Two diploid fusion parents $(2 n=2 x=18)$, Femminello lemon and Valencia sweet orange and a tetraploid somatic hybrid $(\mathrm{SH})$ Valencia $(+)$ Femminello $(2 n=4 x=36)$, were used. The somatic hybrid was produced by polyethylene glycol (PEG)-mediated protoplast fusion of nucellus-derived embryogenic protoplast with nucellar seedling leaf-derived protoplast (Tusa et al., 1990). All the genotypes were grafted onto sour orange $(C$. aurantium L.) and grown at the same location in an experimental field station (Lascari, Palermo, Italy).

For microsporogenesis, floral buds were harvested at various stages of development from early $(2 \mathrm{~mm})$ to nearly full-grown. After removal of petals and calix lobes, the buds were fixed in ethanol-propionic acid (3:1) with a small amount of ferric chloride at room temperature for at least $48 \mathrm{~h}$ and squashed in acetocarmine $2 \%$ onto slides after gentle heating. After coverslip removal by freezing with liquid $\mathrm{N}_{2}$, the slides were made permanent by dipping them twice in butanol-acetic acid (1:1), once in absolute butanol, and finally by mounting the coverslip with Entellan. Pollen stainability and viability were tested by acetocarmine $(2 \%)$ and fluorescein diacetate (FDA), respectively. Pollen germinability was determined on agar medium (2\% agar and 20\% sucrose) at $25^{\circ} \mathrm{C}$ after $36 \mathrm{~h}$.

\section{Results}

Chromosome pairing was examined at diakinesis during microsporogenesis in the tetraploid $\mathrm{SH}$ and its diploid fusion parents (Table 1). SH exhibited multivalent chromosome configurations with, on average, 1.6 quadrivalents per pollen mother cell (PMC) (Fig. 1a). One quadrivalent was found in the donor parent Valencia (Fig. 1b). In the recipient parent Femminello, the mean number of bivalents per PMC was 6.4 , but pairing failure often occurred, giving a high number of univalents (Fig. 1c). In contrast, the occurrence of univalents (mean of 1.1 per PMC) was very low in Valencia. The somatic hybrids $(\mathrm{SH})$ exhibited a range and number of univalents that were intermediate between the parents.

Pollen stainability and viability were higher in $\mathrm{SH}$ than in its parents (Table 2); pollen germinability was $41 \%$ in $\mathrm{SH}$, whereas in the parental lines it varied from $40 \%$ in Femminello to $46 \%$ in Valencia. Variable size characterized pollen grains, fertile and sterile, in both $\mathrm{SH}$ and parents. In $\mathrm{SH}$, the sterile pollen corresponded largely to micropollen grains which were produced by the polyads (Table 2). They predominated among sporads, with a frequency of $66 \%$ in $\mathrm{SH}$, depending on the occurrence of univalents. The unpaired chromosomes showed variable behaviour during microsporogenesis. In $\mathrm{SH}$, they divided at meiosis I (Fig. 1d) or were scattered off the equatorial plate. In the latter case, the lagging chromosomes did not migrate towards the two poles at anaphase I and they were enclosed in an extra nucleus at interkinesis (Fig. 1e). Subsequently, they passed through the second division (Fig. 1f). When the univalents divided during the first meiotic division they formed lagging chromosomes at anaphase/telophase II. At the end of meiosis, the laggards formed two or more micronuclei per PMC in the polyads. Similar behaviour of the univalents was also found in the fusion parent Valencia. In contrast, the univalents in Femminello, which occurred at higher frequencies than in $\mathrm{SH}$, rarely behaved as laggards and generally went to the poles at anaphase I, although the distribution was sometimes asymmetric. However, in both fusion parents, the tetrads were the most frequent type of sporads (Table 2), occasionally containing microspores of different sizes. Moreover, the triads occurred equally in $\mathrm{SH}$ and its parents because of abnormalities in spindle orientation in metaphase/anaphase II (Fig. 1g,h). Meiotic nuclear restitution mechanisms which gave rise to dyads or monads were seen as a consequence of the abnormalities in spindle orientation (Fig. 1i,j).

Table 1 Chromosome associations at diakinesis in meiocytes of Citrus sinensis $(+)$ C. limon somatic hybrids and its fusion parents (Valencia sweet orange and Femminello lemon). Four plants of each genotype were analysed

\begin{tabular}{lccccc}
\hline & & & \multicolumn{3}{c}{ Mean number (range) per diakinesis of } \\
\cline { 4 - 6 } Genotype & Chromosome number $(2 n)$ & Number of diakineses & Univalents & Bivalents & Quadrivalents \\
\hline Valencia & 18 & 25 & 1.1 & 7.1 & 0.8 \\
Femminello & 18 & 21 & $(0-2)$ & $(6-8)$ & $(0-1)$ \\
Somatic hybrid & \multirow{2}{*}{36} & 24 & $(0-18)$ & $(0-9)$ & 0 \\
& & & 2.0 & 13.8 & 1.6 \\
& & & $(0-10)$ & $(9-17)$ & $(1-3)$ \\
\hline
\end{tabular}




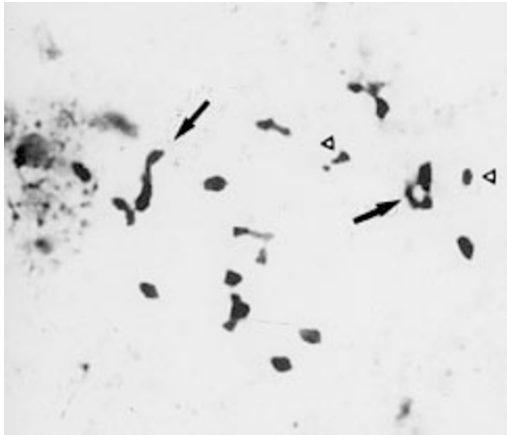

(a)

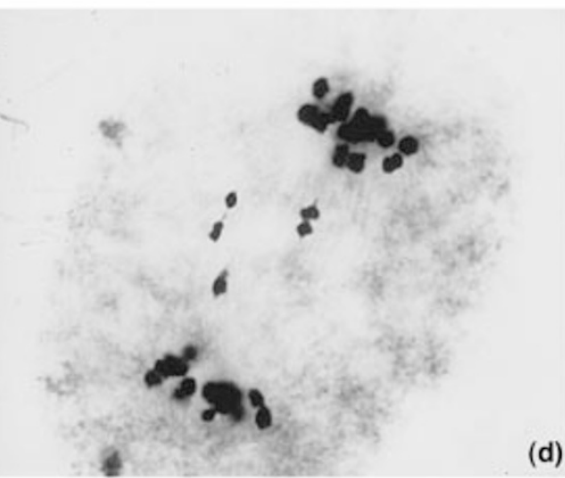

(d)

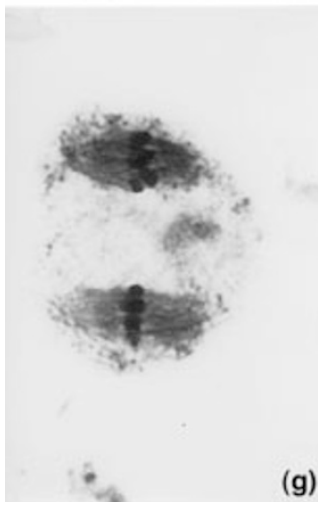

(g)

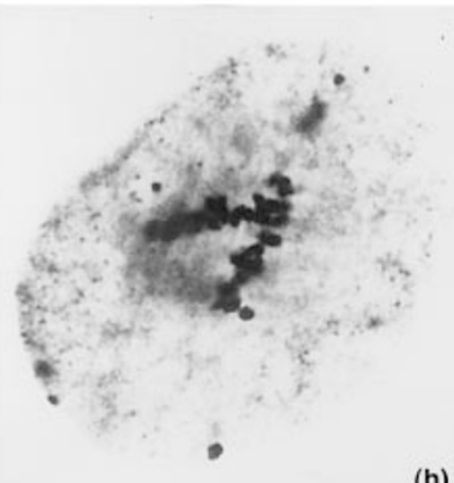

(h)
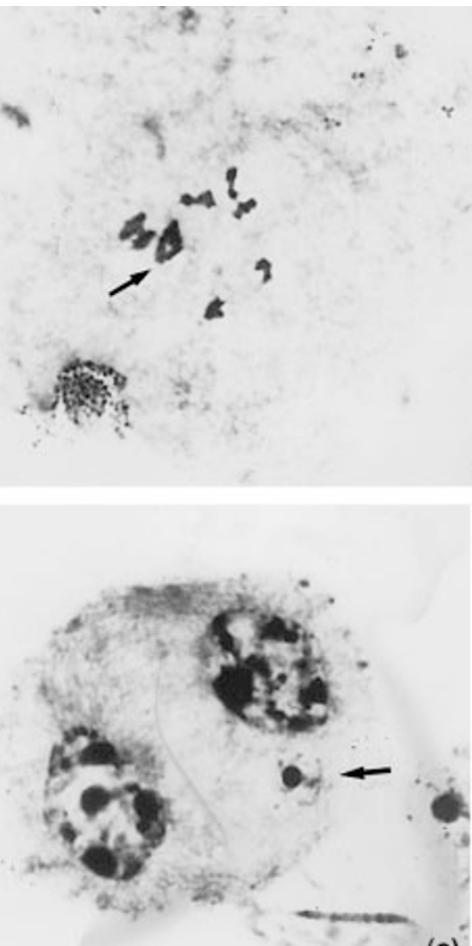

(b)

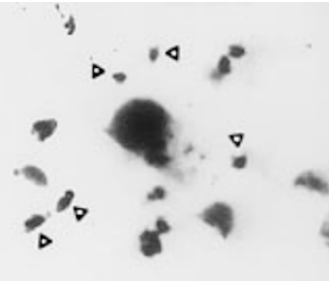

$\nabla$

$\bullet$

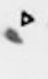

(c)

Fig. 1 Microsporogenesis in the Citrus sinensis $(+)$ C. limon somatic hybrid $(\mathrm{SH})$ and its fusion parents. Chromosome pairing at diakinesis in pollen mother cells (PMCs) showing: (a) 2 I +13 II +2 IV in SH; (b) 7 II +1 IV in $C$. sinensis Valencia; (c) 8 I + $5 \mathrm{II}$ in C. limon Femminello; (d) equational division of univalents at anaphase I in $\mathrm{SH}$; (e) micronucleus (indicated by an arrow) at interkinesis; (f) lagging chromosomes scattered in the cytoplasm at telophase II. Abnormalities in meta-anaphase II and spindle orientation: (g) parallel spindles in C. sinensis Valencia; (h) V-shaped metaphase plates in $\mathrm{SH}$; (i,j) meiotic nuclear restitution events in $\mathrm{SH}(\times 4800)$.

Table 2 Frequency of sporads and pollen stainability in Citrus sinensis $(+)$ C. limon somatic hybrids and its fusion parents (Valencia sweet orange and Femminello lemon). Four plants of each genotype were analysed

\begin{tabular}{lccccccc}
\hline & \multicolumn{6}{c}{ Sporad types (\%) } \\
\cline { 3 - 8 } Genotype & Number of sporads & Monad & Dyad & Triad & Tetrad & Polyad & Pollen stainability (\%) \\
\hline Valencia & 101 & 1 & 0 & 10 & 62 & 27 & 58 \\
Femminello & 157 & 1 & 3 & 8 & 76 & 12 & 51 \\
Somatic hybrid & 110 & 1 & 1 & 10 & 22 & 66 & 64 \\
\hline
\end{tabular}

(C) The Genetical Society of Great Britain, Heredity, 83, 373-377. 


\section{Discussion}

Because of the formation of quadrivalents, gene exchange between Femminello and Valencia occurs in SH. One quadrivalent results from a reciprocal translocation which is present in the Valencia parent and which was also found in different selections by Iwamasa (1969). However, the additional quadrivalents can be considered a consequence of intergenomic pairing. Indeed, good pairing is generally reported between the chromosomes of different Citrus species and between Citrus and its allied genera like Poncirus and Fortunella (Iwamasa, 1969; Raghuvanshi, 1969; Mohammad-Anis \& Raghuvanshi, 1980; Agarwal, 1987).

The behaviour of univalents was variable in $\mathrm{SH}$ and its parents and was similar to that described by Raghuvanshi (1969) in most Citrus species. In SH and Valencia the univalents preferentially formed micronuclei and polyads, whereas in Femminello they were generally enclosed in a nucleus although distributed randomly. In the latter case, the tetrad is the final meiotic product but it contains microspores with an unbalanced chromosome number. The presence of univalents, which is a common feature of Citrus meiosis, is explained by heterozygosity for structural change in chromosomes as well as genetic mutations in synapsis control (Iwamasa, 1969; Raghuvanshi, 1969; Gmitter et al., 1992). The frequency of univalents is negatively correlated with pollen stainability in diploid genotypes of Citrus (Agarwal, 1987; Ibrahim et al., 1994). However, in the tetraploid $\mathrm{SH}$ analysed in this work the pollen stainability and germinability are not low $(64 \%$ and $41 \%$, respectively), even though as many as 10 univalents per cell and a high frequency of polyads occur. A possible explanation for this behaviour is that the chromosomally unbalanced pollen from the tetraploid $\mathrm{SH}$ is viable and able to fertilize. Indeed, different DNA contents were found in the back-cross progeny obtained from diploid Femminello lemon crossed with SH as the pollen parent (Tusa et al., 1996). The nuclear DNA amounts, estimated by laser flow cytometry, ranged from 1.1 to $1.7 \mathrm{pg}$ of DNA, whereas the C. limon nuclear DNA content was $0.8 \mathrm{pg}$. Thus, based on these values, aneuploids, triploids, tetraploids and maybe pentaploids occurred. The presence of tetraploids and pentaploids could be explained through the formation of unreduced eggs in Femminello and unreduced pollen in SH. In the latter, large-sized pollen grains and meiotic abnormalities affecting spindle orientation at meiosis-II and sporad formation are found. Similar cytological features, the most common being parallel spindles, are well known in Solanum where they cause the formation of $2 n$ pollen (Mok \& Peloquin,
1975). In Citrus, $2 n$ egg occurrence is reported in unilateral sexual polyploidization: triploids and tetraploids are obtained from $2 \mathrm{x} \times 2 \mathrm{x}$ and $2 \mathrm{x} \times 4 \mathrm{x}$ crosses, respectively (Esen \& Soost, 1972; Esen et al., 1979). Additionally, tetraploids are obtained from $2 \mathrm{x} \times 2 \mathrm{x}$ crosses by bilateral sexual polyploidization (Russo \& Torrisi, 1951) which supports the formation of $2 n$ pollen in Citrus.

Because the somatic hybrid described in this study is characterized by fertile pollen with different ploidy levels and because it showed intergenomic recombination, it offers interesting possibilities for Citrus breeding programmes for the introgression of useful traits from orange into lemon and for ploidy manipulation.

\section{References}

AGARWAL, P. K. 1987. Cytogenetical investigations in Rutaceae. II. Meiotic studies in three intergeneric hybrids of Citrus. Cytologia, 52, 757-760.

DE JONG, J. H., WOLTERS, A. M. A., KOK, J. M., VERHAAR, H. AND VAN EDEN, J. 1993. Chromosome pairing and potential for intergeneric recombination in some hypotetraploid somatic hybrids of Lycopersicon esculentum ( +) Solanum tuberosum. Genome, 36, 1032-1041.

ESEn, A. ANd soost, R. K. 1972. Aneuploid in Citrus. Am. J. Bot., 59, 473-477.

ESEN, A., SOOST, R. K. AND GERACI, G. 1979. Genetic evidence for the origin of diploid megagametophytes in citrus. J. Hered., 70, 5-8.

GMITTER, F. G. J. R., DENG, X. X. AND HEARN, C. J. 1992. Cytogenetic mechanisms underlying reduced fertility and seedlessness in Citrus. Proc. Int. Soc. Citriculture, 1, 125129.

GRosSER, J. W. AND GMitTer, F. G. J. R. 1990a. Protoplast fusion in Citrus improvement. Pl. Breed. Rev., 8, 339-374.

Grosser, J. W. AND GMITTER, F. G. J. R. 1990b. Somatic hybridization of Citrus with wild relatives for germplasm enhancement and cultivar development. Hort. Sci., 25, 147151.

IBRAHIM, M. M., HELAIL, B. M., ATAWIA, A. A. R. AND SALEH, M. A. 1994. Studies on the chromosomal behaviour and pollen grain viability of some sweet and sour orange cultivars. Ann. Agric. Sci. (Moshtohor), 32, 511-521.

IWAMASA, M. 1969. Chromosome aberration in citrus in relation to sterility and seedlessness. Proc. VIII Int. Citrus Congress, Sun City, South Africa, 1, 175-181.

MOHAMMAD-ANIS, A. AND RAGHUVANSHI, S. S. 1980. Evolutionary significance of chromosomal association in PoncirusCitrus hybrids. J. Cytol. Genet., 15, 166-170.

MOK, D. W. S. AND PELOQUIN, S. J. 1975. Three mechanisms of $2 n$ pollen formation in diploid potatoes. Can. J. Genet. Cytol., 17, 217-225.

PIJNACKER, L. P., FERWERDA, M. A. AND MATTHEIJ, W. M. 1992. Microsporogenesis in three tetraploid somatic hybrids of potato and their di(ha)ploid fusion partners. Theor. Appl. Genet., 85, 269-273.

(c) The Genetical Society of Great Britain, Heredity, 83, 373-377. 
RAGHuvanshi, s. S. 1969. Cytogenetical studies in genus Citrus. IV Evolution in genus Citrus. Cytologia, 27, 172-188.

RUSSO, F. AND TORRISI, M. 1951. Il poliploidismo nei Citrus. Autopoliploidi e allopoliploidi. Ann. Sper. Agr. (Rome), 5, 1041-1062.

SWINGLE, W. T. AND REECE, P. C. 1967. The botany of Citrus and its wild relatives. In: Reuther, W., Batchelor, L. D. and Weber, H. J. (eds) The Citrus Industry, vol. 1, pp. 190-430. University of California Press, Berkeley, CA.
TUSA, N., GRosser, J. W. AND GMITter, F. G. JR 1990. Plant regeneration of 'Valencia' sweet orange, 'Femminello' lemon, and the interspecific somatic hybrid following protoplast fusion. Hort. Sci., 115, 1043-1046.

TUSA, N., FATTA DEL BOSCO, S., NARDI, L. AND LUCRETTI, S. 1996. Obtaining triploid plants by crossing citrus lemon cv. 'Femminello' $2 \mathrm{n} \times 4 \mathrm{n}$ allotetraploid somatic hybrids. Proc. VIII Int. Citrus Congress, Sun City, South Africa, 1, 133136. 